\title{
E-CREDND Scheme for Detection of malevolent nodes in Ad-hoc Network
}

\author{
Shilpi ${ }^{1, *}$, Danish Raza Rizvi ${ }^{1}$ \\ ${ }^{1}$ Department of Computer Engineering, Jamia Millia Islamia, New Delhi-110025, India
}

\section{Abstract}

\begin{abstract}
The remote network that comprises of multipurpose nodes and is infrastructure-less in nature is mobile ad-hoc network (MANET). It powerfully self-composes in subjective and impermanent system topologies. These moveable ad-hoc systems are dishonest and powerless to any interruption in light of their remote association approach. Subsequently, the data from these systems can be stolen effectively just by presenting the aggressor nodes in the system. The hop count metric is calculated to determine the straight route degree. For this reason, directing conventions are arranged. Among all the potential assaults on MANET, identification of wormhole attack is considered to be most problematic. One malevolent node gets packets from a specific area, tunnels them to an alternate transmissible node arranged in another area of the system and deforms the full steering strategy. All routes are merged to the wormhole set up by the aggressors. The total directing framework in MANET gets diverted. Here in this paper, a procedure for identifying malevolent nodes in movable ad-hoc system has been proposed (E-CREDND) and this scheme is critical for the regions where the route separate in the midst of source and goal is two nodes only. This scheme isn't appropriate for those situations where multi-hops are exhibited in the midst of transmitter and recipient. In the anticipated investigation, another procedure is executed for the identification and separation of aggressor sensor nodes from the system. The anticipated plan is used in NS2 and it is portrayed by the propagation results that the anticipated plan demonstrates better execution in correlation with existing methodologies.
\end{abstract}

Keywords: attacker, MANETS, Two phase verification, wormhole

Received on 17 October 2019, accepted on 07 April 2020, published on 09 April 2020

Copyright (C) 2020 Shilpi et al., licensed to EAI. This is an open access article distributed under the terms of the Creative Commons Attribution licence (http://creativecommons.org/licenses/by/3.0/), which permits unlimited use, distribution and reproduction in any medium so long as the original work is properly cited.

doi: 10.4108/eai.13-7-2018.163971

*Corresponding author. Email: july23shilpi@gmail.com

\section{Introduction}

Advances in wireless correspondences, equipment producing innovation, small scale electro-mechanical gadgets and data preparing technologies empowered the improvement of ad-hoc systems. There are without infrastructure and self-building system made out of remotely-connected cell phones. These comprise of various minimal effort, little sensor nodes fuelled by vitality obliged batteries. Figure 1 depicts the basic structure of MANET.

MANETs demonstrates to be a valuable system for different applications, for example, condition observing, medicinal services checking, home machine management and military examinations [1]. They are likewise generally utilized for industry control, management of board and regular citizen life [2, 3]. 
Sensor nodes (SNs) are commonly static while at some point they can be versatile nodes. On the basis of application requirements, they can be conveyed. Base Stations are presented in the system which can either be

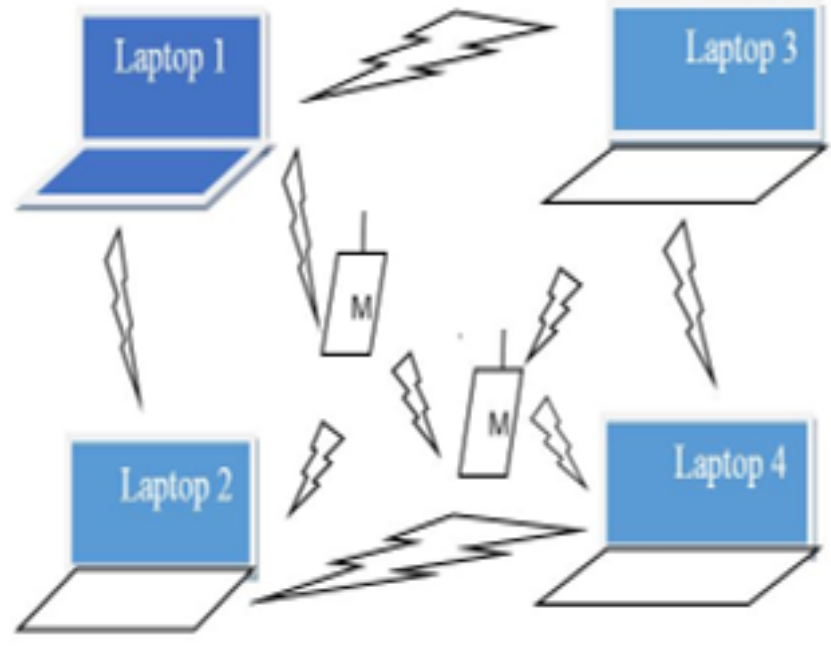

Figure 1: MANET presented diagrammatically

static or versatile. Standard of ad-hoc systems is to accumulate data with the assistance of SNs and sense trial of facts in a specific territory and deliver those readings to base station through multi-hop remote passage. It handled the readings and forwards it to the outer domain via wired or remote connections. It fills in as portal in the middle of the deployed system and the outside world $[4,5]$.

In SN, vitality utilization has notable effect on systems' lifespan. A few vitality sparing advancements have been proposed, for example, energy-efficient MAC, cycle planning, vitality gathering, energy recharging, node substitution and energy balance. As correspondence in sensor systems consumes great power, along these lines a proficient directing convention to adjust the vitality utilization among sensor nodes is required [6]. It needs to drag out the system lifetime just as give intends to better information transmission.

Ad-hoc systems have a few attributes that make them powerless against different assaults in assorted and threatening conditions:

- Sensor nodes are asset obliged. They have constrained memory, vitality, registering force, data transfer capacity and correspondence variety.

- Arrangement of nodes in encourages aggressors to dispatch different sorts of assaults.

- Network topology is dynamic. It is conveyed in unfriendly condition coming up short on any fixed framework. Accordingly, consistent reconnaissance of the system is troublesome.

- Security conventions that are strong can debase the execution of applications as it costs more assets. In this manner an exchange off must be set among execution and the security.

- Everyone has a wireless system channel. Anyone can use a radio designed for comparable recurrence band to partake or screen the channel correspondences. In this way, aggressors can helpfully break into the ad-hoc systems.

As an outcome of previously mentioned attributes, these ad-hoc systems are extremely fragile to numerous assaults [7, 8]. Wormhole attacks are one of the most serious and danger to MANETs directing conventions, where malevolent nodes are put deliberately to contort the topology of system and tunnel packets specifically utilizing wrong settled routes. It is challenging to recognize inconsistencies or wormhole attacks for the reason that ad-hoc systems are unconventional.

The wormhole attacks can be carried out by the inner nodes (traded off nodes inside the system that process packages simply like typical nodes) or outside nodes (who just send packages and do not practice the cryptographic) [9], of which the inner aggressors are progressively risky and hard to recognize [10]. The incredible works of writing give over the top consideration to the outer wormholes however disregard interior wormholes that are additionally usual in MANETs.

A case of this is the interconnection among different apparatuses drawing nearer from comparable district like an organization meeting for shaping an ad-hoc system amid the accessibility of system administrations. After the gathering of any data, the mobile nodes will send the data further to their neighbourhood nodes [11]. The data sent by the middle person nodes will continue as a switch while some node requires to advance data to a portable node that it is past the territory of sensor node correspondence. The accomplishment of resolute routes for exchanging data isn't feasible due to the arbitrary development of SNs. The inaccessibility of any fixed infrastructure is one of the fundamental issues related to this system. In this system, the sensor nodes have the skill to act as both router and host.

The real application is they can self-design themselves which is used for provisioning correspondence, for example, in disaster inclined regions. They are exceptionally valuable in those regions where network is stuck because of natural causes. Both static and dynamic topology is utilized in MANET directing conventions.

Along these lines, a procedure that can distinguish malevolent node from the system is required. We propose E-CREDND, a novel calculation for wormhole identification and confinement, which can identify wormholes without extra equipment. We test the correctness of our novel calculation by reenacting and contrasting it and other wormhole identification calculation: CREDND [12], who likewise utilizes hop distinction and neighborhood checking. The results prove that our method outclasses existing method.

The paper is composed as: Section 2 discovers the researches already given for wormhole identification and prevention. Then in 3rd section we have considered wormhole attack and its effects on system. We state the issue in section 4. In 5th section thorough introduction of projected plan, its algorithm and flowchart have been 
offered. Section 6, the aftereffects and their assessment has been revealed. At last, section 7 accomplished the paper and emphasized the significance of wormhole identification in network.

\section{Related Work}

X. Luo et.al, anticipated a convention in making a Credible Neighbor Discovery in contradiction of wormholes in remote systems that distinguish outside wormholes through the hop difference and inner wormholes by empowering the mutual fellow nodes as observers to screen if the confirmation packages are forded by malevolent nodes. The anticipated calculation is basic, localized convention and does not need any extraordinary equipment, localization or synchronization. [12] have considered wormhole recognition under nondeterministic arrangement and furthermore anticipated the idea of neighbor ratio threshold to figure out which all nodes should be distinguished, which adds to improving the precision and vitality productivity of wormhole location. However, this arrangement can't function admirably in the state that all nodes with various correspondence ranges, progressively changing and adjusting to different circulations.

S. Majumder et.al, proposed a calculation on Absolute Deviation (AD) of measurable way to maintain a calculated space from and antedate wormhole assault. Absolute Deviation Covariance and Correlation set aside not so much effort to recognize this assault than previously established [13]. Any settings such as GPS is not required in proposed calculations. The calculations proposed are light in weight and calculation costs is additionally truncated. Toughness, chief aspect of factual methodology. Preferred execution by AD strategy over AODV is demonstrated by simulation in MATLAB test system for wormhole assault. It has been seen that proposed calculation is $73 \%$ more proficient than traditional one and less influenced by outliers. The package drop design is additionally estimated for wormholes utilizing Absolute Deviation Correlation Coefficient.

J. Padmanabhan et. al, suggested a versatile and dispersed plan which utilized consecutive likelihood proportion test to evade single point disappointments and to deal with high portability, with no extra asset prerequisites [14]. Framework overheads regarding correspondence, calculation and capacity viewpoints are registered and impact of framework parameters on the suggested plan have been examined. It was discovered that for assumed estimations of framework parameters, wormholes were recognized utilizing couple of packets and for frameworks including exceptionally versatile nodes, the arrangement is much better as far as quicker location times. All recreations were performed utilizing Omnet++.
D. S. K. Tiruvakadu et.al, expressed that numerous arrangements have been given to shield MANET from assaults, yet these arrangements are probably going to recognize numerous bogus assaults as genuine. Similar false assaults happen frequently when an assault is intricate and they have many highlights as like a wormhole assault. Along these lines, [15] exhibited Wormhole Attack Confirmation (WAC) framework utilizing honeypots to alleviate incorrect cautions in adhoc system and ensure its assets. Affirmation of assault directs the need of breaking down the potential situations of an assault. So as to break down this assault, they have manufactured a Wormhole Attack Tree (WAT) in view of its side effects. The honeypot affirms the wormhole assault utilizing the WAT and history of assaults. The tested outcomes have shown that the expressed arrangement is proficient in affirming wormhole attack, subsequently sparing assets and limiting the way refoundation.

Jianpo Li et. al, presented Distance vector-hop (DVhop), a localisation calculation which frequently endures the wormhole attack. [16] proposed a DV-hop localisation calculation in case of wormhole assault (AWDV-hop). Every one of the node get record of their neighbor nodes through neighbor node relationship list (NNRL). The nodes that are suspicious can be found by looking at the hypothetical and genuine number of neighbor nodes. At that point, the presume signal nodes figure the distance to further nodes present in their NNRL to figure out the confirmed assaulted nodes. For the purpose of analysis, the different area marked with 1 or 2 for the identification of mentioned nodes. In the following round, the nodes marked 1 and the nodes marked 2 disengaged from one another. The reenactment effects demonstrate that the localisation error of the AWDV-hop calculation is diminished approximately by $80 \%$ when compared to DV-hop calculation contrasted with secure neighbor discovery based DV-HOP (NDDV-hop) and label-based DV-HOP (LBDV-hop) calculation, the localisation error is decreased by around $34 \%$ and $7 \%$, separately.

RanuShukla et.al, offered a trust based secure steering conventions to deal with compelled nature of WSN and there is back and forth in Security and Energy. [18] improved Trust and Energy aware Secure Routing Protocol (TESRP) by verifying it counter to wormhole assault. It is a standout amongst the finest trust-based convention however this convention doesn't give safety from wormhole assault. It demonstrated execution improvement as far as remaining vitality, throughput, and system lifetime as contrast with other trust-based conventions. It gives better results since it can distinguish defective nodes just as noxious nodes though others can't. The offered convention doesn't require time synchronization or geographic data, it accomplishes adjusted vitality utilization. So, here trust calculation along sequence number idea is utilized in verifying TESRP from wormhole assault.

Kavitha $\mathrm{T}$ et.al, stated that issue due to portability of nodes in versatile specially appointed system is 
connection letdown. [19] recommended an Instant Route Migration (IRM) convention, which develops the way momentarily considering the way separation and hop tally. IRM convention incorporates essential directing table that contains the briefest way from source to goal. What's more, it incorporates another backup steering table for route support if there should be an occurrence of connection letdown. The past works identified with connection letdown and re-steering experiences delay in conveying packets. Be that as it may, the recommended arrangement accomplishes most extreme throughput, less start to finish delay and gives moment course relocation than existing frameworks. Reserving system and optional table component helps in quick re-steering. Likewise, in recommended arrangement, the connection status is observed routinely.

Bharat Bhushan et.al, articulated the utilizations of the remote ad-hoc. In [20] sensor systems are conveyed in the threatening situations for detecting the physical conditions. These systems have the open correspondence because of which it experiences greater security concerns. [20] presented the worry for the wormhole attack as it corrupts the usefulness of entire system. Alongside, they talked about the instrument for wormhole recognition and resistance based on acquired area data and time synchronization between nodes in the system. Here, [20] additionally examined about packet leashes, another path for wormhole attack location and resistance. These are intended to shield single remote transmission from wormholes. There are two kinds of leashes: temporal and geographical leashes. It has been seen that temporal leashes are more powerful than geographical leashes as they do not require communicate affirmation and can be used for systems in which time synchronization is cultivated successfully.

H. Ghayvat et.al, showed the secure way to deal with wormhole distinguish and alleviate. [21] verified AODV which can productively discover wormhole attack and consumed Digital Signature to counteract it. This methodology depends on an estimation of time taken by passage to break down the conduct of wormhole. A short time later, it sets some static limit esteem. In view of this time and limit esteem, it chooses if node is malevolent/wormhole node or reliable node. Then two calculations are connected to alleviate the wormhole node: digital signature and hash chain. The established conclusions have exhibited that showed methodology has expanded lifetime, throughput and limits organize deferral of the versatile system when contrasted with existing arrangements. It gives QoS upto an attractive dimension yet evacuation of undesirable mistakes in wormhole recognition is as yet an issue to address.

S. K. Jangir et.al, demonstrated a deliberate audit completed on the condition of the exploration outcomes on wormhole assaults. The mimicked conclusions evaluated the relative exhibitions of the various arrangements proposed. Different strategies and methods utilized for the position and aversion of wormhole assaults, for example, package leashes, indication receiving wires, time sensitive systems and some more. A nearby report has been done on different conventions (like OLSR, DSR and AODV) and attacks. It is hard to state that one arrangement is appropriate in all circumstances. However, utilizing various methodologies anticipated in [22], a more grounded discovery system can be conceived for forestalling wormhole attack in systems.

Chitra Gupta et.al, indicated a method which found an elective route to the goal node. For this, the thickness and versatility of nodes is checked. The execution of the safe route disclosure convention is achieved utilizing NS2 and by alteration of the AODV directing convention. This method depends on specific variables like packet conveyance proportion, throughput, steering overhead drop, etc. [23].

\section{Wormhole Attack}

One of the utmost demanding assaults possible in MANET is wormhole attack. In this, two malevolent nodes build a wormhole tunnel. These malevolent nodes directs data of received packages towards another side of system through passage and after that they replay that data. Since the system structure and the correspondence respectability are not thrashed, the wormhole attack is hard to distinguish [28]. It is sometimes referred as tunnelling attack.

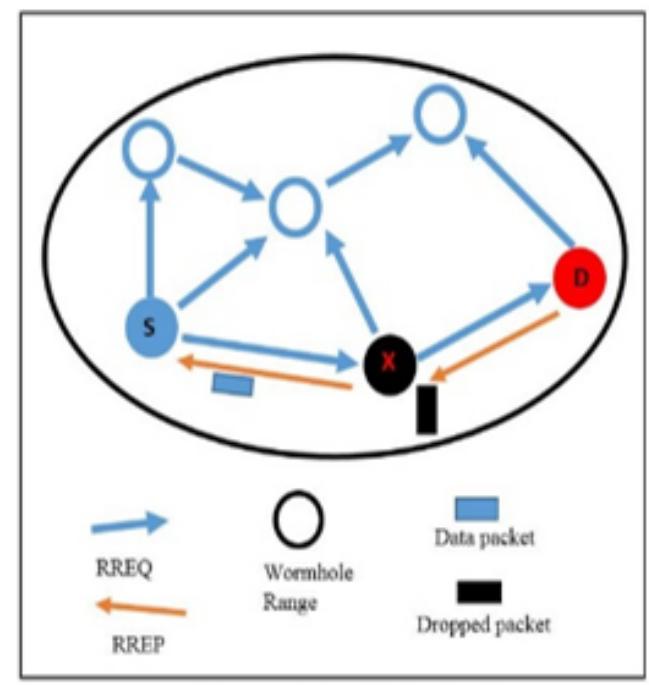

Figure 2: Wormhole Attack

Figure 2 demonstrate that aggressor node in black color, with great broadcast power, covers source node in blue color and goal which is in red color and turned out to be the most limited middle of the road node to achieve goal D. The investigation of assault arrangement explored that; attacker dependably goes for dipping packages rather to divide content. In this manner, malevolent node or action can't be recognized by any cryptographic or conventional security arrangements [29]. The MAC convention information isn't required inside this attack 
and the cryptographic strategies also have no impact on it. Consequently, end of such attack in the system is troublesome.

\subsection{Wormhole Attack Types}

Subsequent are types of wormhole attacks that produce depending on the number of nodes included and the manner in which it occurs:

- Wormhole attack utilizing Encapsulation or Inband Channel: Only a legitimate path is used to route each of the package when a wormhole end obtains it. In order to prevent the nodes from rising of hop tallies, the legitimate path gets encapsulated here. The second end point generates original form of the package once again $[30,31]$.

- Wormhole attack utilizing Out-of-Band Channel: For generation of a wormhole link, a keen out-ofband high data capacity station is produced amongst the end points in the two-ended wormhole attack $[32,33]$.

- Figure 3, representation of above two types of wormhole attack [25]. Source S (Blue) is directing package to goal D (Red) where two malevolent nodes attack the system and re-directs or drop the packet. Sometimes, these malevolent nodes can change the contents of the packages.

- Wormhole attack utilizing Packet Relay: There is a necessity to provide one malevolent node such that amongst two nodes that are at distance, the packages can be replayed. Thus, the generation of fake neighbors is done in this manner. It is also called "replay-based attack".

- Wormhole attack with High Power Transmission: Here, malevolent node has limited technique for high-control. Any node that builds up the ground-breaking pass, rebroadcasts the RREQ in the direction of the goal. By this manner, the chance of malevolent node grows to be in the routes in the middle of source and goal $[30,31]$.

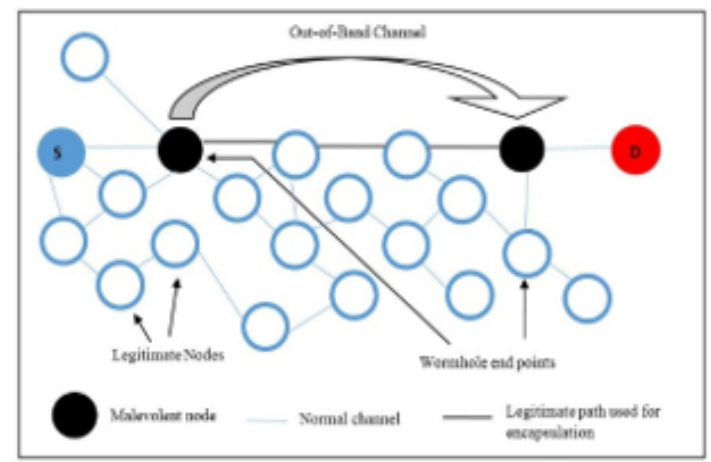

Figure 3: Types of Wormhole AttackEncapsulation and Out-of-band

\subsection{Models of Wormhole Attack}

There are three different models generated due to the packet accelerating behaviour of wormhole end points and the mechanism through which the identities can be hidden or shown in the network. The source and goal are included in each of these models along with the malevolent nodes or attackers as shown in the diagrams below.

- Open Wormhole Attack: The observing of RREQ packets of the system is done within an open wormhole attack due to the presence of malevolent nodes [26]. The malevolent nodes are assumed to be available on another path by the other hop in the system. Thus, the identity of both nodes is easily seen in the system. Figure 4 below show this model:

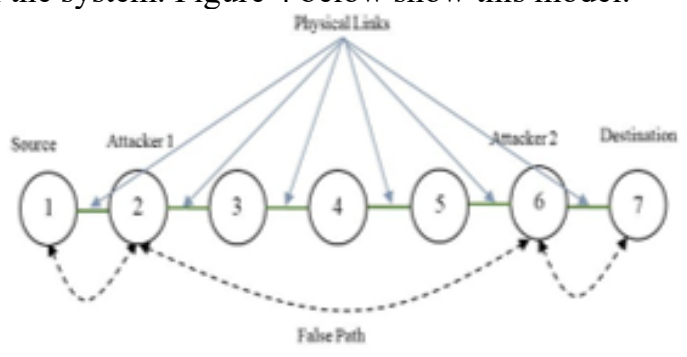

Figure 4: Open Wormhole Attack

- Half-Open Wormhole: This attack is very similar to above one. The only difference is that the identity of only one node is shown here and another node is unseen from the system. Thus, only at one end, the modification of packets will be done as shown in the Figure 5.

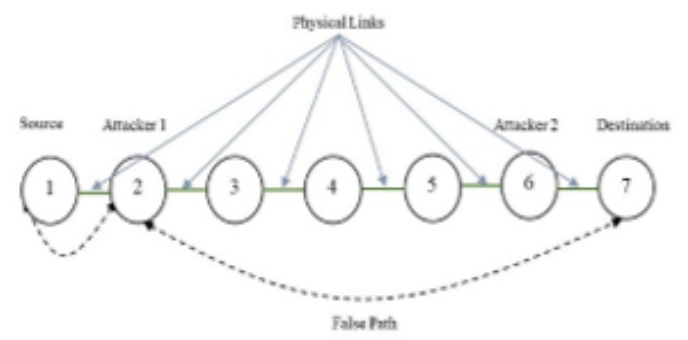

Figure 5: Half-Open Wormhole Attack

- Closed Wormhole: On trail from source to goal, the identities of all the in-between nodes are unseen. Only one-hop of distance is assumed to be present in amid source and goal within this situation. Therefore, the generation of fake neighbors is done here. It is depicted in Figure 6. 


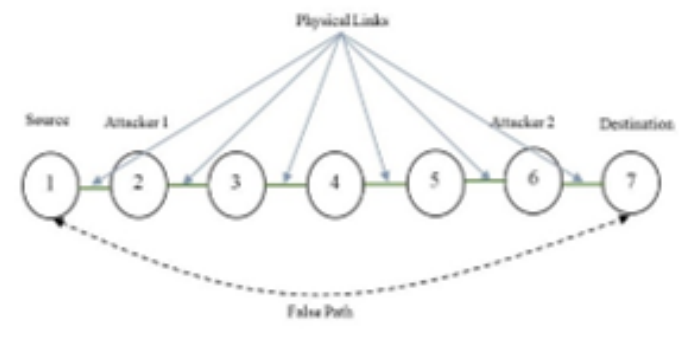

Figure 6: Closed Wormhole Attack

\subsection{Another Classification}

Another classification against wormhole attack is given by Bharat Bhushan et.al [20]:

- Proactive countermeasures anticipate wormholes by usage of specific equipment for time estimation or exact time synchronization or for power transmission in a specific bearing. Packet leash can be utilized in such proactive countermeasures for wormholes which is utilized for single-bounce secure pair-wise time synchronization.

- Reactive countermeasures don't forestall the wormhole arrangement. These can't stay away from wormholes in the event that it is utilized for uninvolved attack. Hence to shield against uninvolved attacks, a few strategies, for example, mysterious interchanges can be utilized.

\subsection{Strategies to oppose Wormhole Attack}

- Node exclusion:

- Modified DV-hop calculation [34] - It locks every one of the nodes which are continuing the wormhole assault and incorporate few ordinary nodes, to take out the impact of the wormhole assault on the system.

- Symmetric code encryption based DV-HOP (SDV-hop) [35] - It bars the signal node, whose error surpasses as far as possible, to oppose the wormhole assault. Nonetheless, it will miss roughly reference point nodes, which lead to the asset dissipate.

- Hardware correction:

○ SenLeash [36] - depends on the node included indicating receiving wire. It forbids the compromised node by guessing the transmitting bearing of signal.

○ Round Trip Time (RTT) [37, 38, 39] - is grounded on the nodes having clock synchronization. They can distinguish and prohibit the condemned steering way by looking at broadcast time and the normal time distinction.
- Correction mechanism: This strategy changed the hops between attacked signal nodes [40, 41]. It joins the DV-hop calculation and outline in opposition of wormhole assault, yet it is a basic strategy which has restricted aftereffects on opposing wormhole assault.

The very first discussed strategy can totally dispose of the impact of the wormhole assault, however countless normal nodes are lost. The second discussed strategy needs support of extra equipment, which will expand the expenses of system. The third discussed system has low unpredictability also, low-vitality utilization, however it can't totally dispense with the impacts of wormhole attack. So, [16] proposed a security DV-hop localisation calculation against wormhole assault.

\section{Problem Statement}

MANET, the distributed kind of system where adaptable nodes can leave or join the system when they need. In such sort of system, security, directing and nature of administration are the serious issues which influence system's performance. The essential goal of MANET is to find the route. For maintaining routes between nodes is taken care by directing conventions in MANET. The assurance of the proficiency of a directing convention is by expending the battery control by directing of traffic into the system. Severe restrictions are imposed on directing conventions due to high dynamic nature of system. Hence, we have used ad-hoc on-demand vector (AODV) as directing convention, which builds way from source to goal in least amount of time and furthermore maintain the administration nature in the system. Besides, AODV is not based on any safety mechanisms. So, an impersonation attack can be effortlessly done. Wormhole attack is one of them. It is the dynamic kind of assault which influences system performance to unbelievable degree. It is activated by the malevolent nodes which make tunnels in the system and raise delay in the system. The different procedures by different authors have been structured to distinguish malevolent nodes which raised delay and degraded the performance of whole system as these malevolent nodes drop all the packets. The procedures which are structured so far either require additional equipment or programming for the location of malevolent nodes. The methods which are proposed so far are likewise founded on the edge-based systems for the recognition of malevolent nodes. The edge estimation of specific parameters can change because of particular variables like congestion or connection failure. At the point when the limit estimations of parameters shift which influence exactness of noxious node identification. The methodology is required which does not depend on additional equipment or programming for the malevolent node identification and confinement from the system. 


\section{Research Methodology}

Wormhole attack, a dynamic sort of assault where delay is increased in the system. The idleness of the system get expanded at consistent rate when delay is expanded i.e. both the factors are proportional to each other. The proposed process depends on the identification of malevolent nodes which are capable to generate wormhole attack in the system and has two noteworthy stages for the identification of malevolent nodes.

Stage 1: In the first stage, the source node and goal nodes are characterized in the system. The source node floods the system with route request packages. Then it computes the round-trip time (RTT) of the route solicitation and route reply messages in the system. While computing RTT, the source node begin clock when flood route demand messages and notice the time for every node when get route reply parcels. The source keeps up the rundown of every node for the route solicitation and reply RTT. The source chooses the best way from source to goal dependent on the hop tally and grouping number.

Stage 2: In the second stage, the malevolent nodes get identified from the system. The source node begins transmitting information over the chosen way from source to goal (Stage 1). Then it computes the time of information parcels at each hop until reach goal. The time of each hop is contrasted with the RTT of route reply parcels. The node which possess critical high energy for information parcel transmission are set apart as the malevolent nodes in the system. To separate malevolent nodes from the system, method of multipath steering is connected in the system. In the multipath steering, when the malevolent nodes exit that way will be disregarded in the system.

\subsection{Proposed Algorithm}

Input: Mobile Nodes

Output: Detection of Malevolent nodes

Begin

1. Deploy wireless ad hoc network of adaptable nodes with finite number

2. State source and goal nodes in the network

3. Path establishment

3.1. If the path exits between source and goal

\subsubsection{Start data broadcast}

3.2. Else

3.2.1. Source flood route request packages in the system

3.2.2. Source start timer to notice route reply time
3.2.3. Source maintain list of each node with the time when receive route reply message at source

3.2.4. Best way was selected by source till goal

3.2.5. Way having least hop tally and most extreme sequence number will be picked in step 3.2.4

4. Malevolent node Detection

4.1. The source start transmitting data over the selected path

4.2. The source notice data packet arrival time at each hop

4.3. If node has significant high time than route reply time

4.3.1 Mark node as malevolent 4.4. Else

4.4.1 Source transmit data over selected path

4.4.2. Repeat step 4 until data broadcast get completed

End

\subsection{Proposed Flowchart}

\begin{tabular}{|c|c|}
\hline Parameters & Values \\
\hline Simulator & NS2-2.35 \\
\hline Area & $800 * 800$ \\
\hline Number of nodes & 24 \\
\hline Antenna type & Omi-directional \\
\hline Queue type & Priority queue \\
\hline Queue length & 50 \\
\hline Propagation model & Two ray \\
\hline Table 1: Simulation parameters \\
\hline
\end{tabular}




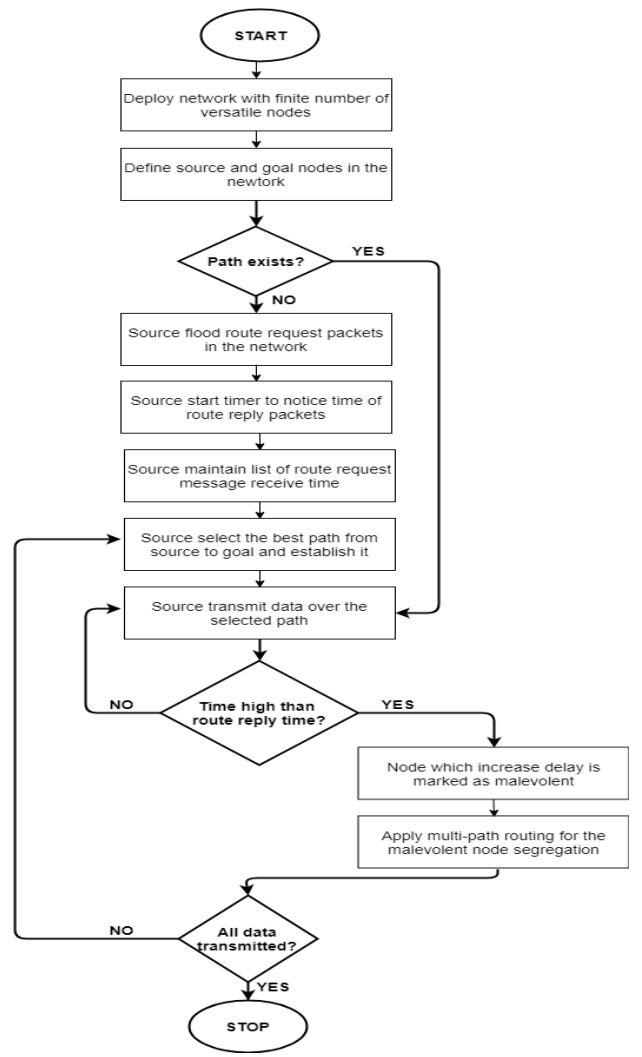

Figure 7: Proposed Flowchart

\section{Experimental Results}

This examination work is acknowledged with identification and separation of malevolent nodes from versatile ad-hoc system. Here, two situations are implemented and looked at. In the first situation, existing strategy that is CREDND [12] for the identification of malevolent is implemented, which likewise utilizes hop distinction and neighborhood checking to spot wormholes. In the proposed outline, the two-phase verification scheme is implemented for the recognition of malevolent nodes in the system, E-CREDND. The execution of projected strategy is contrasted concerning packet loss, delay and throughput which is displayed in this section of paper. The Table 1 demonstrates the simulation parameters.

\subsection{Packet Loss}

Figure 8 depicts the results of proposed two phase verification (E-CREDND) and CREDND scheme in terms of packet loss for the identification of malevolent nodes. It is studied that packet loss of former is low as compared to latter. The third scenario which is compared is attack scenario and it is analyzed attack scenario has maximum packet loss as compared to both CREDND and ECREDND schemes.

\subsection{Delay}

In Figure 9, it is analyzed that the delay of the ECREDND scheme is low which is shown with the blue line as contrasted to CREDND scheme which is shown with green color for the malevolent node identification in the system. The delay of the attack scenario is shown with the red color which is maximum as compared to CREDND and E-CREDND situations.

\subsection{Throughput}

As shown in Figure 10, the throughput of the E-CREDND technique is compared with the CREDND scheme for the malevolent node identification. It is detected that throughput of the E-CREDND technique is quite high as compared to CREDND technique. The throughput proves reliability of the E-CREDND technique as compared to CREDND technique. The throughput of the attack situation is minimum as contrasted to E-CREDND and CREDND techniques.

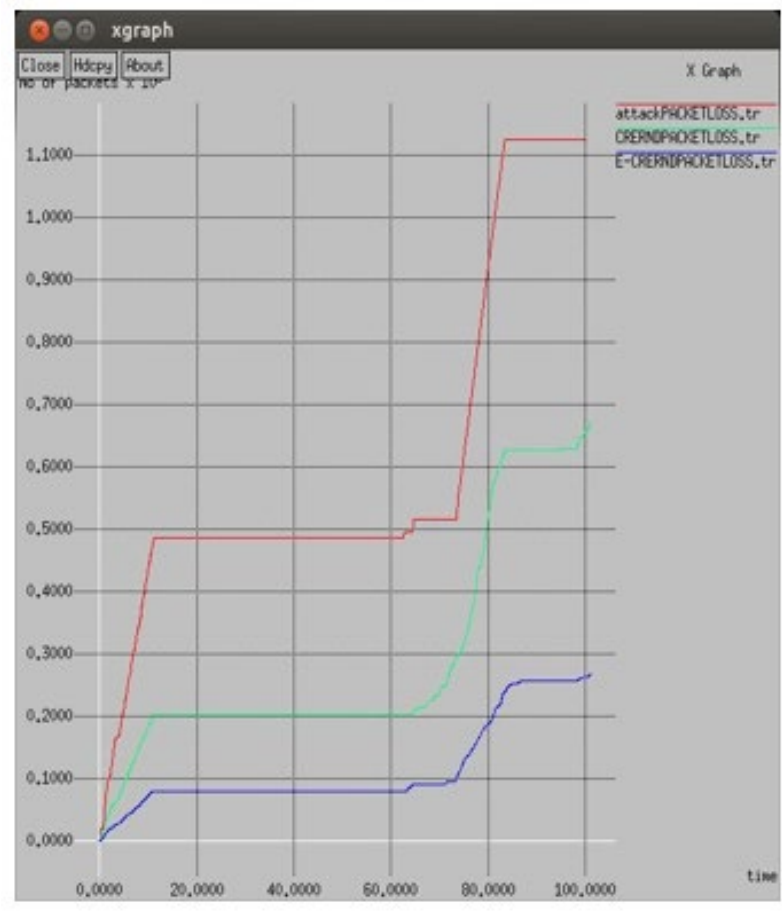

Figure 8: Packet Loss comparison 


\section{Conclusion}

It is recognized that the remote ad-hoc frameworks are dispersed sort of systems in which versatile nodes can join together or withdraw the framework as per them. No center controller is displayed. System security, direction finding and service quality are the fundamental issues because of the confidence character of the framework. A dynamic sort of assault named wormhole attack might be the reason of the entering of attacker nodes in the framework and as a result of this delay increments. In the offered research, two phase verification is used. For the acknowledgment of attacker versatile nodes, this strategy demonstrates less precision and extensive execution times. The anticipated and open methodologies are applied in NS2 and the results delineate improvement in throughput, decrease in delay and packet loss.

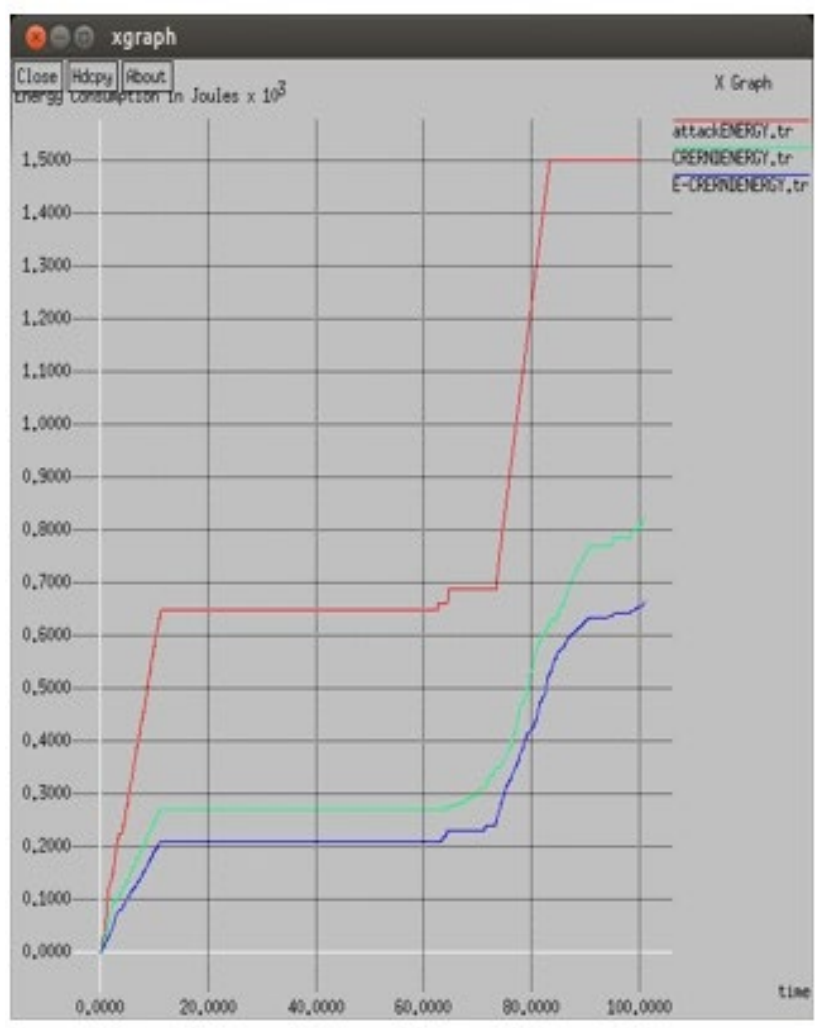

Figure 9: Delay comparison

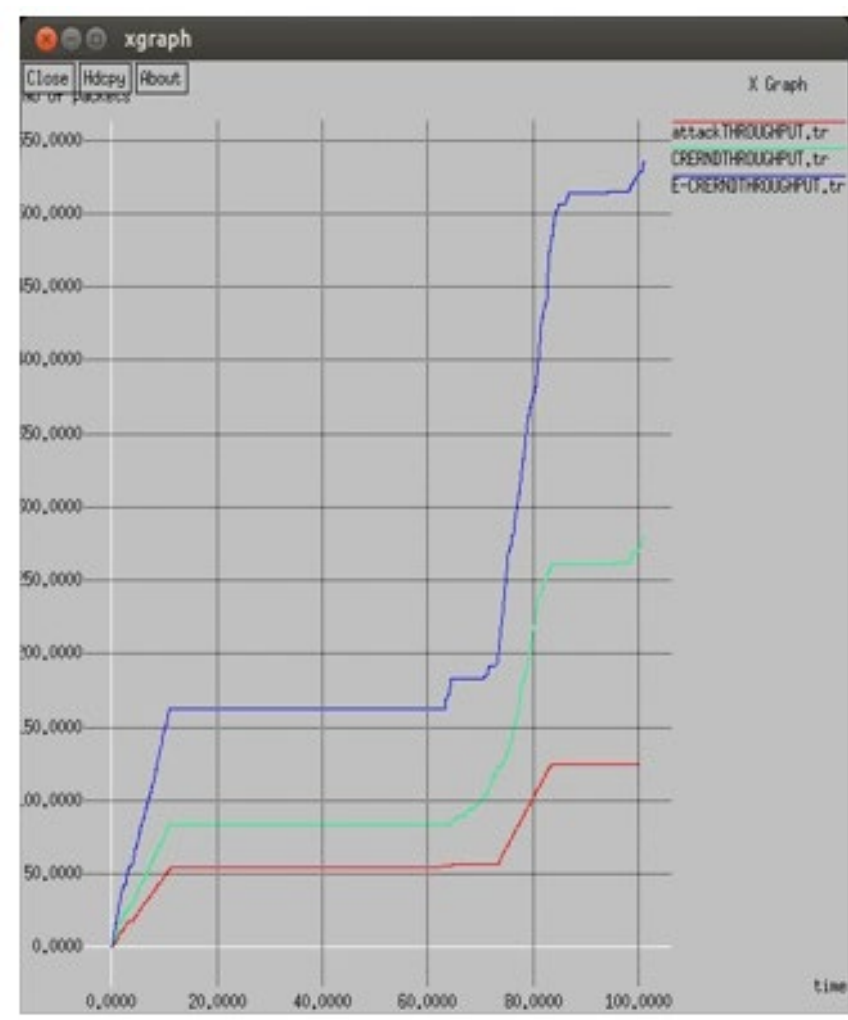

Figure 10: Throughput comparison

\section{References}

[1] A. Ajith Kumar S., Knut Ovsthus and Lars M. Kristensen, "An Industrial Perspective on Wireless Sensor Networks A Survey of Requirements, Protocols and Challenges", IEEE Communications Surveys \&n Tutorials, Vol. 16, No. 3, Third Quarter 2014

[2] J. Luo, D. Wu, C. Pan and J. Zha, "Optimal energy Strategy for Node Selection and Data relay in WSN-based IoT", Mobile Networks and Applications, Vol. 20, No. 2, April 2015

[3] J. Chen, X. Cao, P. Cheng, Y. Xiao and Y. Sun, "Distributed Collaborative control for Industrial automation with wireless sensor and actuator networks", IEEE Transactions on Industrial Electronics, Vol. 57, No. 12, December 2010

[4] A. Nosratinia, T.E. Hunter and A. Hedayat, "Cooperative communication in wireless networks", IEEE Communications Magazine, Vol. 42, No. 10, 2004

[5] Srdjan C., Levente B. and Jean-Pierre H., "SECTOR: Secure Tracking of Node Encounters in Multi-hop Wireless Networks", ACM Workshop on Security of Ad Hoc and Sensor Networks (SASN), October 2003.

[6] S. T. Tanwar, N. K. Kumar and J. R. Rodrigues, "A Systematic review on heterogeneous routing protocols for wireless sensor network", Elsevier Journal of Network and Computer Applications, Vol. 53, No. 1, July 2015

[7] Q. Yang, X. Zhu, H. Fu and X. Che, "Survey of security technologies on wireless sensor networks", Journal of sensor, Vol. 2015, Article ID 842392, December 2015

[8] B. Bhushan and G. Sahoo, "Recent Advances in Attacks, Technical Challenges, Vulnerabilities and Their 
Countermeasures in Wireless Sensor Networks", Wireless Personal Communications, Vol. 98, No. 2, January 2018.

[9] G. Kumar, M. K. Rai, and R. Saha, "Securing range free localization against wormhole attack using distance estimation and maximum likelihood estimation in Wireless Sensor Networks", Journal of Network and Computer Applications, Vol. 99, December 2017

[10] F. A. Khan, M. Imran, H. Abbas, and M. H. Durad, "A detection and prevention system against collaborative attacks in mobile ad hoc networks", Future Generation of Computer Systems, Vol. 68, March 2017

[11] VK Sagtani, and SKumar, "Modern Approach to Enhance Routing Recitation in MANET". International Journal of Emerging Technology and Advanced Engineering, Vol. 4, No. 7, July 2014

[12] X. Luo, Y. Chen, M. Li, Q. Luo, K. Xue, S. Liu and L. Chen, "CREDND: A Novel Secure Neighbor Discovery Algorithm for Wormhole Attack", IEEE Access, Vol. 7, January 2019

[13] S. Majumder, Prof. Dr. D. Bhattacharyya, "Mitigating Wormhole Attack in MANET Using Absolute Deviation Statistical Approach", IEEE 8th Annual Computing and Commination Workshop \& Conference (CCWC), January 2018

[14] J. Padmanabhan and V. Manickavasagam, "Scalable and Distributed Analysis on wormhole links in Wireless Sensor Networks for Networked Systems", IEEE Access, Vol. 8, February 2018

[15] D. S. K. Tiruvakadu and V. Pallapa, "Confirmation of wormhole attack in MANETs using honeypot", Computers and Security, Vol. 76, July 2018

[16] Jianpo Li, Dong Wang and Yanjiao Wang, "Security DVhop localisation algorithm against wormhole attack in wireless sensor network", IET Wireless Sensor Systems, Vol. 8, No. 2, March 2018

[17] Pratik Gite, "Link Stability Prediction for Mobile Ad-hoc Network Route Stability", International Conference on Inventive Systems and Control, October 2017

[18] RanuShukla, Rekha Jain, P. D. Vyavahare, "Combating against Wormhole Attack in Trust and Energy Aware Secure Routing Protocol (TESRP) in Wireless Sensor Network", Proceeding International conference on Recent Innovations is Signal Processing and Embedded Systems (RISE -2017), October 2017

[19] Kavitha T, Muthaiah R, "Instant Route Migration during Link Failure In MANETS", International Journal of Mechanical Engineering and Technology (IJMET) Vol. 8, No. 8, August 2017

[20] Bharat Bhushan and Dr. G. Sahoo, "Detection and Defense Mechanisms against Wormhole Attacks in Wireless Sensor Networks", 3rd International Conference on Advances in Computing, Communication \& Automation (ICACCA), April 2017

[21] H. Ghayvat, S. Pandya, S. Shah, S. C. Mukhopadhyay, M. H. Yap and K. H. Wandra, "Advanced AODV Approach For Efficient Detection And Mitigation Of WORMHOLE Attack IN MANET", Tenth International Conference on Sensing Technology, IEEE, December, 2016

[22] S. K. Jangir and Naveen Hemrajani, "A Comprehensive Review on Detection of Wormhole Attack In MANET", International Conference on ICT in Business Industry \& Government (ICTBIG), IEEE, April 2016

[23] Chitra Gupta and Priya Pathak, "Movement Based or Neighbor Based Technique for Preventing Wormhole Attack in MANET", Symposium on Colossal Data Analysis and Networking (CDAN), IEEE, September 2016
[24] Pallavi Sharma and Prof. Aditya Trivedi, "An approach to defend against wormhole attack in ad hoc network using Digital Signature", IEEE 3rd International Conference on Commination Software and Networks, September 2011

[25] S. Gupta, S. Kar and S. Dharmaraja, "WHOP: Wormhole Attack Detection Protocol using Hound Packet", International Conference of Innovations in Information Technology, 2011

[26] T. Hayajneh, P. Krishnamurthy and D. Tipper, "SECUND: A Protocol for SECUre NeighborhooD Creation in Wireless Ad hoc Networks," 5th International Conference on Collaborative Computing: Networking, Applications and Work sharing, Vol.1, No. 2-3, January 2009

[27] S. Khurana and N. Gupta, "FEEPVR: First End-to- End protocol to Secure Ad hoc Networks with variable ranges against Wormhole Attacks", 2nd International Conference on Emerging Security Information, Systems and Technologies, IEEE, September 2008

[28] J. Li, X. Zhong and C. Xu, "Review of dynamic node localization algorithm for wireless sensor networks", Journal of Northeast Dianli University, Vol. 35, No. 1, 2015

[29] Mayank Kumar Sharma and Brijendra Kumar Joshi, “A Mitigation Technique for High Transmission Power based Wormhole Attack in Wireless Sensor Networks", International Conference on ICT in Business Industry \& Government (ICTBIG), IEEE, April 2016

[30] M. Jain and H. Kandwal, "A survey on complex Wormhole Attack in wireless Ad Hoc Networks", In the International Conference on Advances in Computing, Control and Telecommunication Technologies, IEEE, January 2009

[31] M. Azer, S.E. Kassas and M.E. Soudani, "A Full Image of the Wormhole Attacks :Towards Introducing Complex Wormhole Attacks in wireless Ad Hoc Networks", International Journal of Computer Science and Information Security, Vol. 1, No. 1, May 2009

[32] U.K. Chaurasia and Varsha Singh, "MAODV: Modified wormhole detection AODV protocol", 6th International Conference on Contemporary Computing (IC3), IEEE, September 2013

[33] Z. A. Khan and M. H. Islam, "Wormhole Attack: A new detection technique", In the International Conference on Emerging Technologies, IEEE, December 2012

[34] H. L. Chen, W. Lou, Z. Wang, "Securing DV-hop localization against wormhole attacks in wireless sensor networks", Pervasive Mobile Computing, January 2015

[35] H. B. Wang, L. P. Feng, R. Li, "The secure localization algorithm of SDV-Hop in wireless sensor networks", Telkomnika Telecommunication Computer Electronic Control, Vol. 1, No. 3, 2016

[36] R. H. Hu, S. M. Dong, "SenLeash: a restricted defense mechanism against wormhole attacks in wireless sensor network", J. Communication, Vol. 34, No. 10, October 2013

[37] P. Amish, V. B. Vaghela, "Detection and prevention of wormhole attack in wireless sensor network using AOMDV protocol", Proceedings of International Conference Communication, Computing and Virtualization (ICCCV), Vol. 79, February 2016

[38] N. Agrawal, N. Mishra, "RTT based wormhole detection using NS-3", Proceedings of International Conference on Computational Intelligence and Communication Networks, November 2014

[39] S. Subha, U. G. Sankar, "Message authentication and wormhole detection mechanism in wireless sensor 
network", IEEE 9th International Conference on Intelligent Systems and Control (ISCO), January 2015

[40] Q. M. Zhou, Y. He, "Simulation of wormhole attack in SDV-hop and its resistance method", Computer Engineering Applications, Vol. 25, No. 46, 2010

[41] H. Zhou, W. Zhou, "An improved DV-hop algorithm based on detection of wormhole attack", Digital Technology Applications, Vol. 3, No. 2, 2014

[42] Koppisetti Giridhar, C. Anbuananth and N. Krishnaraj, "Research on Various Routing Techniques in Wireless Adhoc Networks", International Journal of Recent Technology and Engineering (IJRTE), ISSN: 2277-3878, Vol. 8, Issue-1S4, June 2019

[43] Wael Y Alghamdi, Mohsen Rezvani, Hui Wu and Salil S Kanhere, "Routing-Aware and Malicious Node Detection in a Concealed Data Aggregation for WSNs", ACM Transactions on Sensor Networks (TOSN), Vol. 15, No. 2, April 2019

[44] Wei She, Qi Liu, Zhao Tian, Jian-Sen Chen, Bo Wang and Wei Liu, "Blockchain Trust Model for Malicious Node Detection In Wireless Sensor Networks", SPECIAL SECTION ON MOBILE SERVICE COMPUTING WITH INTERNET OF THINGS, IEEE Access, April 2019

[45] Xueqiang Yin and Shining Li, "Trust evaluation model with entropy-based weight assignment for malicious node's detection in wireless sensor networks", EURASIP Journal on Wireless Communications and Networking, 2019 